17. The ganglia are the nervous centres of the vessels, as the spinal marrow is the nervous centre of the entire muscular system.

18. The ganglionic influence supplied to the vessels acts by a kind of attraction for some or all parts of the contained fluid; thus being the antagonist to the contractility of the coats of the vessels. It is not impossible that muscular nerves may excite contraction by precisely similar power, the difference in result arising from difference in the sphere of influence of the terminations; the muscular nerve acting amongst muscular fibres; the vascular nerve acting on or through the lining coat of the vessel. In this case, the nervous energy would be one and the same, differently distributed for convenience of operation.

19. This ganglionic influence is no more essential to circulation, nutrition, or secretion, than the spinal influence is to muscular contraction; but by regulating the quantity of fluid in a given part, according to its requirements, or other circumstances, it has a powerful mediate influence over all the functions of life.

20. Ganglia are never the centres of muscular motion. All the reflex action through them-if reflex it can be called-is the supply of nervous energy to vessels in answer to impressions of condition conveyed from the vessels themselves, or from the surface on which they are distributed.

21. These impressions of the existing condition (e. g., of the stomach and duodenum during the different stages of digestion) do not normally give rise to sensation, but to what may be termed ganglionic perception; in answer to which an increased flow of nervous energy, occasioning an increased supply of blood when and where it is required, ensues.

22. As correctness and acuteness of sensation are intimately connected with the circulation in the part, and as sensation is not directly subject to the will, every sensation probably is attended with some modification of the small vessels of the part affected For this reason, all sensory nerves are ganglionic nerves also.

23. As the uncertain, irregular, and unequal operations of the mind forbid any regularity or constancy in acts purely voluntary; if motor nerves when passing through ganglia can affect the action of the ganglia; it is obviously desirable that no purely voluntary muscular nerve should pass through a ganglion.

24. But as muscular acts of an involuntary kind are performed with regularity, and are governed by the existing state of the surface with which they are functionally connected, it is plausible enough to suppose that every supply of spinal energy to the involuntary muscle should correspond with a supply of ganglionic energy to the vessels and surfaces with which the muscle cooperates. Hence involuntary muscular nerves do pass through ganglia; and wherever a muscular nerve does pass through a ganglion, there will always be found a physiological association between the parts supplied by the nerves from that ganglion.

25. The ganglia are the centres of sympathetic disorder of the vessels, but not of sensations or muscular movements.

26. It is probable that the action of the ganglionic, as of other parts of the nervous system, is to a certain extent vicarious and compensatory; so that if one ganglion is exhausted, its nerves may receive energy from other ganglia, and if one portion of the system is greatly overtaxed, it thus weakens for the time all the rest. Restricting the word sympathy to the organic processes, the great sympathetic nerve is rightly named, being in this way the grand associator of the many different parts of the organic system.

May 20th, 1845 .

\section{ON THE TREATMENT OF GANGRENA SENILIS.}

By Henry S. TAYLor, Esq. Surgeon, Guildford.

The communication on Gangrena Senilis in The I.ANCET of last week, calls in question the claims of certain eminent men of the present day to originality in their treatment of that disease, although it does not appear that they ever arrogated it to themselves. A perusal of this and other publications on the same subject, has led me to conclude that many of the greatest authorities, at home as well as abroad, attach undue importance to the constitutional treatment; and the local nature of the disease seems to have been lost sight of altogether in the endeavour to redress that general irritation of the system which is only consecutive. If we are to accept the explanation given by Carswell of this pathological state - viz, that it is dependent on obliteration of the arteries in the limb, and this on disease of the arterial tissue, it is not to be expected that medicinal agents would exert much influence over it, any more than in other instances of senile degeneration.

It follows from this view, that the main indication should be to preserve the capillary circulation of the part in sufficient activity. That this is to be done in some measure by general treatment, I am not prepared to deny, but it will be more certainly effected by preventing any loss of temperature in the extreme parts by surrounding them with some non-conducting substance. I allude now to the method recommended by Sir B. Brodie, in his Lectures on Mortification, ${ }^{*}$ which does not appear to have excited all the attention it deserves.

In one of these admirable discourses he speaks most favourably of the practice of wrapping the affected limb in carded wool, as pursued at Greenwich Hospital. 'This has also been adverted to by Mr. S. Cooper, in one of the later editions of his "First Lines."

It is the more singular that these simple means should have been overlooked, when we consider the usual course of the symptoms - that there is great coldness of the extremity as the first signal of approaching mischief, that during the spread of the gangrenous process the affected parts are often represented as being at a lower temperature than the room, and that it is only when the line of demarcation has declared itself that the contiguous parts recover their vital heat. Again: in the treatment of aneurisms after the performance of Hunter's operation, a somewhat analogous case, this principle is kept in view carefully enough, and we are well admonished to lose no time in enveloping the limb in flannels \&c., while its temperature is being watched with the utmost solicitude.

I am encouraged to offer these remarks by the experience of a few cases of this form of gangrene, where the above plan was adhered to; and in one of these the success of the treatment was so unexpected, that I venture to subjoin a brief account of it:-

An old man, a small farmer in this neighbourhood, hale and very active, although in his 74 th year, came to consult me, Sept. 14th, 1844, for what he called gout in one of his feet. He stated that for four or five days the great toe and the next one to it had been very red, as if inflamed, and felt burning hot, particularly when in bed, or whenever the leg was elevated. On examination, I found these toes to be very red, with a purplish tint, which was deepest at their tips and around the nails; the parts were, however, decidedly colder than elsewhere, though he maintained that they were much hotter to him. The pulse could not be felt in the tibial arteries, nor very distinctly in the popliteal; the limb generally was not hot nor swollen, and no pain was felt as long as he was about on it. As to his general health, he said he was never better in his life. Suspecting what was to be the nature of his attack, I urged him to go home to bed, and to keep his foot and leg warm with flannels; at the same time I gave him a pill, of calomel one grain, opium half a grain, to take every night, and a lead lotion, to be used warm, as a placebo.

September 17 th.-He came to me again, having neglected all my advice. The toes at first affected were now quite purple, very cold to the touch, and the surrounding parts were red and tumid to above the ankle. I again impressed upon him and his wife-who, by the way, was a young woman-the necessity of his keeping in bed; and I gave them instructions to get lambswool in the rough state, and having wrapped his foot and leg in a thick layer of it, to sew the whole up together in flannel. The dose of opium was increased to one grain every night, and ten drops of the tincture were given three times a day in bitter infusion.

20th.-On paying him a visit I found him in bed, and upon unswathing the limb, the great toe seemed to be quite recovered, and only the last joint of the other retained any livid hue; the parts were also quite warm, and the sensation of burning had left. him.

October 5th.- Since the last report the old fellow persisted in going about again, having discarded the wool, and wearing only a list slipper. The two last joints of the second toe are now quite black and covered with phlyctenæ, and are cold and devoid of feeling. He has been rambling out of his bed at night, and talking incoherently; he is languid, out of spirits, and very irritable. He was persuaded to return to bed, and to resume the ervelopes of wool and flannel. I prescribed for him carbonate of. ammonia and tincture of calumba with fifteen drops of laudanum every six hours; to take a grain of opium at night; and his appetite being good, his usual diet, which was a liberal one, to be continued.

20th.-On removing the coverings from his foot at this visit, I found the gangrene had stopped, and the last joint was the only. part which was completely dead; there is some commencement of suppuration; and his nights are passed in tranquil sleep, and his manner is more composed.

25th.-Suppuration is now fairly established, the ulcer is dressed with oiled linen, to prevent the wool adhering. The opium is now given at night only.

From this period separation proceeded very slowly, and it was quite the end of December before $I$ was able to remove the small 
piece of mortified substance; this consisted of only so much of the last phalanx as supports the nail ; the resulting sore was equally slow in filling up. As the foot was still colder than the other, I directed him to wear thick woollen stockings, and a shoe wadded with the wool, during the remaining winter months.

It may be objected, with regard to this case, that the opium, which was exhibited for a long time, had the greatest share in the cure. If so, the success was far beyond what is usually realized by this medicine; and the effects of the local treatment were too obvious to require any comment. In conclusion, I would state my conviction that if this patient had been treated with the usual applications, whether the general treatment were phlogistic or antiphlogistic, he would only have recovered with very serious mutilation.

June 27 th, 1845 .

\section{CONTRIBUTIONS IN MIDWIFERY.}

By J. Halc Davis, M.D., Physician to the Royal Maternity Charity.

Difficult parturition, from large size and unusual ossification of child's head, complicated ly retention of urine, the distended bladder reaching nearly to the umbilicus-Puerperal convulsions apprehended-Treatment-Remarks.

I WAS requested by a medical friend, at half-past five one Tuesday morning in October last, to come to his assistance in a case of difficult labour, upon which he had been more or less in attendance during the previous forty-eight hours. It was a first preguancy, and the patient's age was eight and thirty. The liquor amnii had escaped at 10 P.M. on the previous Saturday; sligh bearing pains had occurred at long intervals during that nigh and the following day, (Sunday;) but the os uteri had dilated very slowly, being thick and rigid.

The patient during next day (Monday) was very feverish; the parts had hecome hot and dry, the os tince, the orifice of which, in the morning, had reached its present diameter, (three-fourths of full dilatation, had now become greatly swollen as to its anterior segment, more rigid even than before, and tensely applied to the head's surface. The patient had vomited quantities of green bile. On these accounts my friend had taken blood from the arm, to the amount of sixteen ounces, and with the effect of considerably diminishing the urgency of the patient's distress. At 4 P.M., the patient felt a strong call to pass water, but could not respond to it; the catheter was introduced, and twelve ounces of urine were drawn off. Within the last few hours she has again suffered from an urgent inclination to pass her urine without the power, and the peculiar swelling of a distended bladder is apparent to the taxis, extending upwards upon the gravid uterus to within a short distance of the umbilicus. My friend has endeavoured to relieve his patient again by the use of the metal catheter, but without success. On making the attempt myself, althongh I applied considerable pressure by my fingers on the head of the child upwards and backwards, with the view of taking off its firm bearing upon the neck of the bladder, (a mancuvre frequently successful,) I likewise was foiled. I then requested my friend to procure me a gum-elastic male catheter with the stilette in it, and with a little humouring of this instrument, I accomplished my object, removing an amount of thirty ounces, much to the relief of the patient.

There had been strong pains, and no lack of them during the whole of Monday, and the previous Sunday night, and up to the time of my summons on Tuesday morning, but without any progress having been effected. The head was perfectly wedged in the brim of the pelvis, completely immovable, hence the fruitlessness of the attempt which had been made prior to using the catheter, to take off its pressure upon the neck of the bladder. Added to the distended bladder, I found in the case the following points deserving of attention:-The anterior segment of the os tince swollen, hard, closely and tightly capping the head, over which there was a considerable protrusion of scalp; the parts hot, intensely so; the skin hot and dry, the pulse hard and full; the patient restless, with turgid face, and an expression of countenance peculiarly expressive of distress; the pains violent, yet ineffectual. Under such an aspect of the case, especially in a first labour, where the tendency to convulsions is so great, the amount of resistance to the agents of labour being so extreme, and the distress and irriability of the patient so marked, there could be no two opinions as to the propriety of a further abstraction of blood; the constitution and habit of the patient, moreover, offered no contraindication, she being of firm fibre, and moderate en bon point. A vein was opened in the arm, eighteen ounces of blood withdrawn, when the pulse became soft, and the skin moist; but the rigidity of the os uteri remained the same, or nearly so; no difference in its character could be perceived. For a few minutes after the bleeding, the pains lessened somewhat in their violence, bat soon returned of their former strength, and with no better effect than before; the patient, however, expressed herself thankful for much relief afforded her.

I now determined upon delivery by embryotomy. In the performance of that operation, a free use of the osteotomist became necessary, as the head was unusually ossified. Considerable care was observed, and time taken, in the operation, on account of the pelvis being of considerable depth, the head, therefore, being at a proportionally greater distance from the outlet, as well as from the circumstance of my having to act within a circle of such small dimensions as the only partially dilated uterine orifice allowed me. Little force of traction being used at any one time, (the use of the osteotomist rendering it unnecessary, the delivery was at length accomplished in perfect safety, and the patient did not complain of any aggravation of her sufferings during its progress.

From the condition of the funis, I should judge that the child might have been dead eight or ten hours. The greater portion of the placenta was urged by the expulsive efforts of the uterus into the upper part of the vagina, but a spasmodic contraction of the neck of the uterus upon the remainder, proved a cause of retention; the mass was, however, removed entire without diffculty. The womb contracted firmly, and there was no hæmorrhage. Sixty drops of tincture of opium were exhibited within an hour after delivery, contrary to my usual practice, on account of some disposition to nervous excitement; and one-eighth of a grain of tartar emetic, in dilute liquor ammoniæ acetatis, to be taken every three hours, was prescribed.

Directions were left that the room should be darkened, and that our patient should be kept perfectly quiet, and in a moderate temperature. Pulse 100, but soft.

Visit, 7 P.M. - The patient has had three hours' sleep. She feels comfortable; has no pain nor tenderness of abdomen; head entirely free from any uneasiness; skin of natural temperature; pulse 70 , and soft; tongue furred, but less so than during the labour; no thirst; has rot passed water. To be visited by her medical attendant again at ten, with a view to the employment of the catheter, if necessary.

Visit, 1 P.M. Wednesday.-The patient has had a good night's rest; skin cool; pulse 64, and soft; tongue now quite clean. She passed water last night at half-past nine, and without any difficulty, in a full stream, so that the catheter was not required. Has no pain nor tenderness anywhere. Ordered, castor oil, six drachms.

Nothing was observed but steady improvement in any subsequent visits, and $I$ have been since informed by the attendant, that his patient had been enabled to go out of doors with impunity within the month, with his permission.

Remarks.-There are several points worthy of remark in the ahove case. The patient was in labour for the first time, at an advanced age, the tissues on that account were likely to be less succulent and yielding than they are wont to be at a much earlier period of life. Had this circumstance alone existed, the labour, in all probability, would have been protracted, although it might not have proved instrumental. There was, however, in addition, the presentation of an unusually ossified head of a large child the capacity of the pelvis, if it fell short of what is considered standard, did so but in a trifling degree, it was relatively small, not absolutely. The pelvis was, however, deeper than is deemed normal, by one inch and a half behind, as measured from the promontory of the sacrum to its tip. This circumstance rendered the delivery a little more tedious, as also did the circumscribed space left by the nterine aperture in which to operate. The more than ordinary resistance to the agents of labour, with the usual amount of plethora present in the system of a pregnant and parturient female, determined the febrile commotion of the system in this case. The disturbance of the nervous function, generally greater in primipara, constituting in them a predisposition to puerperal convulsions, was thus much aggravated, as well as by another circumstance, the distended state of the bladder, a source of irritation of itself (more particularly in one parturient for the first time) sufficient to expose the patient to an attack of that disease. The presentation of the head, which experience tells us corstitute another predisposition to the above convulsions, its firm impaction, and pressure against very sensitive tissues, rendered, as a measure of safety to the subject of the above case, the several parts in the treatment indicated, highly important.

The first abstraction of blood, practised before I saw the patient, was judiciously determined upon; the only difference I would have suggested would have been, in addition, the employment of tartarized antimony, in one-sixth and one-eighth of a grain doses, with the view of exciting nausea, relaxing the tissues, and to supersede, if pcsible, the necessity for a further abstraction of 